\title{
Effect of Voltage Rise Rate on Streamer Branching and Shock Wave Characteristics in Supercritical Carbon Dioxide
}

\author{
Tomohiro Furusato, Member IEEE, Miyuki Ota, Tomoyuki Fujishima, \\ Takahiko Yamashita, Member IEEE, Takashi Sakugawa, Member IEEE, \\ Sunao Katsuki, Member IEEE, and Hidenori Akiyama Fellow, IEEE
}

\begin{abstract}
This paper reports on dependence of voltage rise rate on positive streamer branching and shock waves in supercritical carbon dioxide. Voltages with rise rates of 0.21 and 4.5 $\mathrm{kV} / \mathrm{ns}$ were applied to a needle-to-plane electrode. Pre-breakdown phenomena involving streamer growth and shock wave propagation were observed by means of a shadowgraph method. Results show that the spread angle of the streamer at the needle tip under $4.5 \mathrm{kV} / \mathrm{ns}$ was nearly twice as large as that under $0.21 \mathrm{kV} / \mathrm{ns}$. The discharge may initiate without density reduction due to electrostrictive force. While shock wave Mach number was little affected by the voltage rise rate, it increased by increasing the negative voltage peak. Consequently, the velocity of shock wave is presumed to be influenced by population of the vibrational state of carbon dioxide.
\end{abstract}

Index Terms - Nanosecond pulsed discharge, positive streamer, shock waves, supercritical carbon dioxide, shadowgraph method, electrostrictive force.

\section{INTRODUCTION}

$\mathrm{D}$ ISCHARGE plasma in supercritical (SC) fluid displays excellent controllability of both physical properties and chemical reactivity and is thus anticipated in new chemistry synthesis fields such as phenol polymerization [1], metallic oxide nanomaterials [2], carbon nanomaterials [3] and nanodiamonds [4]. Recently, the study of a pulsed power switch using SC-CO ${ }_{2}$ and $\mathrm{SC}-\mathrm{N}_{2}$ has been launched as a novel application [5]-[7]. Understanding of streamer discharge and shock waves in SC fluids is of importance in the development of a $\mathrm{SC}$ reactor.

Several studies related to direct current (DC) breakdown characteristics have been reported. For example, in SC-He, DC breakdown voltage was seen to drop in the vicinity of the critical point under the quite narrow gap of $3 \mu \mathrm{m}$ under a

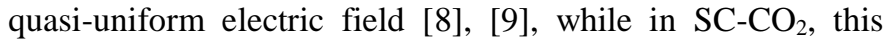

The manuscript was submitted in March 2016 "This work was supported by Grant-in-Aid for Research Activity Start-up (No. 26889048) and by TEPCO Memorial Foundation".

T. Furusato, T. Fujishima, and T. Yamashita are with the Graduate School of Engineering, Nagasaki University, Nagasaki 852-8521, Japan (t-furusato@nagasaki-u.ac.jp).

M. Ota, T. Sakugawa, S. Katsuki and H. Akiyama are with the Graduate School of Science and Technology, Kumamoto University, Kumamoto 860-8555, Japan (e-mail: m.ota@st.cs.kumamoto-u.ac.jp; sakugawa@cs.kumamoto-u.ac.jp; katsuki@cs.kumamoto-u.ac.jp; akiyama@cs.kumamoto-u.ac.jp). critical anomaly did not occur under gaps ranging from 80 to $200 \mu \mathrm{m}$ under a non-uniform electric field [10], [11]. Despite these great discrepancies, little attention has been given to the study of pre-breakdown phenomena in SC fluids.

No unified pre-breakdown theory in dense medium yet exists due to its complex mechanism, with both the direct impact ionization model and bubble theory independently proposed through many studies [12], [13]. However, recent research has suggested a new pre-breakdown mechanism: ultra-short pulsed voltage in dielectric liquids [14]-[16], which is that nano-sized pores created by the electrostrictive force in the vicinity of the needle tip affects the pre-breakdown mechanism in dielectric liquids with pulse widths ranging from sub-nanosecond to 13 nanoseconds.

Thus far, the authors have studied pre-breakdown mechanism in $\mathrm{SC}-\mathrm{CO}_{2}$ with pulsed voltage widths of a few hundred nanoseconds. The results showed that the generation of initial electrons under negative polarity was supposed to field emission [17]; direct impact ionization mechanism was supposed by back-discharge analysis under positive and negative polarity [18], [19].

This study investigates positive streamer branching and shock wave characteristics as a function of voltage rise rate while taking into account the electrostrictive force so as to elucidate initiation processes under $\mathrm{SC}-\mathrm{CO}_{2}$ with ultra-short pulsed voltage.

\section{EXPERIMENTAL SETUP AND PROCEDURE}

\section{A. Discharge observation system and experimental condition}

The shadowgraph observation system is shown in Fig. 1. The experimental system consisted of a high pressure chamber, a pulsed power generator, shadowgraph system (Nd: YAG laser and a digital CCD Camera (Nikon D60)) and a delay-pulse generator for synchronous operation between the voltage source and shadowgraph system. The single-flash laser has a 5 ns pulse duration and $12 \mathrm{~mJ}$ energy, and its output is frequency doubled $(\lambda=532 \mathrm{~nm})$. The shadowgraph image with $5 \mathrm{~ns}$ time resolution is obtained while opening the CCD camera for $1 \mathrm{~s}$. Two kinds of pulsed power generators were used to compare the voltage rise rate. The topology of the pulsed power circuit is described in section II-B. The needle electrode was composed of tungsten. The gap length was set at $1 \mathrm{~mm}$ and the radius of 


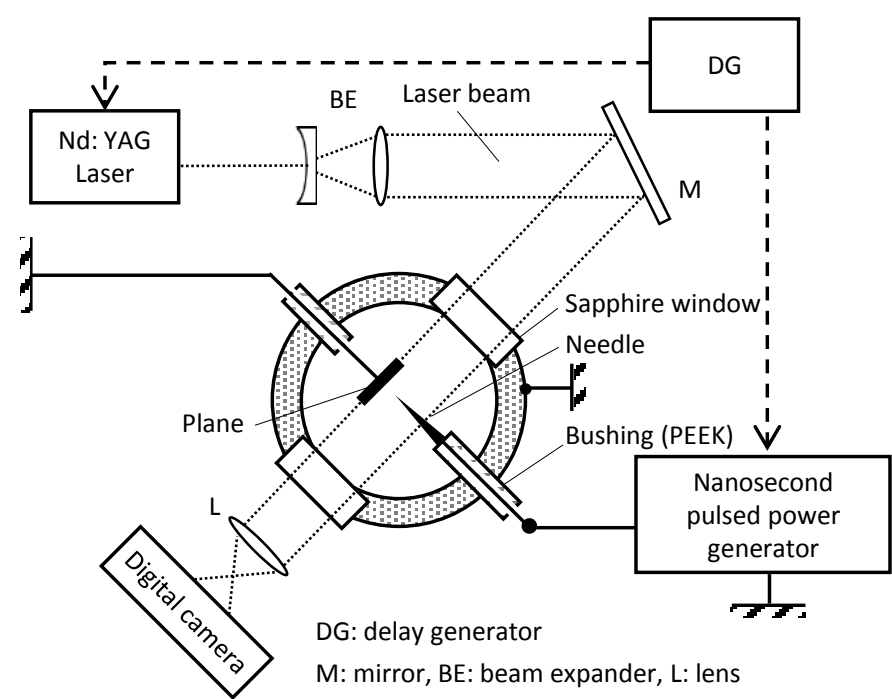

Fig. 1. Diagrammatic illustration of shadowgraph observation system.

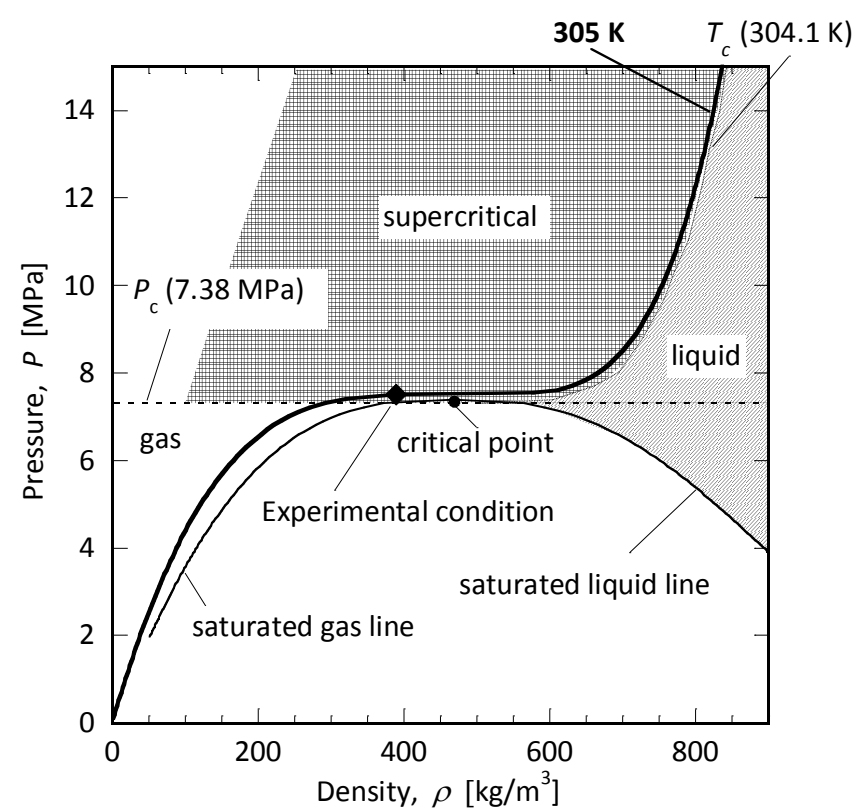

Fig. 2. Illustration of phase diagram of carbon dioxide. Experimental condition $(T=305 \mathrm{~K}, P=7.5 \mathrm{MPa})$.

the needle tip was $0.5 \pm 0.1 \mu \mathrm{m}$. A phase diagram of $\mathrm{CO}_{2}$ is illustrated in Fig. 2. High purity $\mathrm{CO}_{2}$ (purity: $>99.990 \%$ ) was injected into the high pressure chamber. $\mathrm{CO}_{2}$ temperature was controlled by a temperature controller with heater bar and thermocouple, with test condition under SC phase $(T=305 \mathrm{~K}$, $P=7.5 \mathrm{MPa})$.

\section{B. Pulsed power generator}

The secondary circuit of the inductive pulsed power generator (IPPG) is shown in Fig. 3. The forward current first flowed through a fast recovery diode (FRD: Voltage Multipliers Inc. Z100FF3). The pulsed voltage was generated by discontinuing the FRD reverse current simultaneously with saturation of saturable transformer $(\mathrm{ST}) . \mathrm{L}_{1}(0.63 \mu \mathrm{H})$ and $\mathrm{L}_{2}$ $(0.54 \mu \mathrm{H})$ are the inductance of the saturated ST and of the line of load circuit, respectively. The following voltage

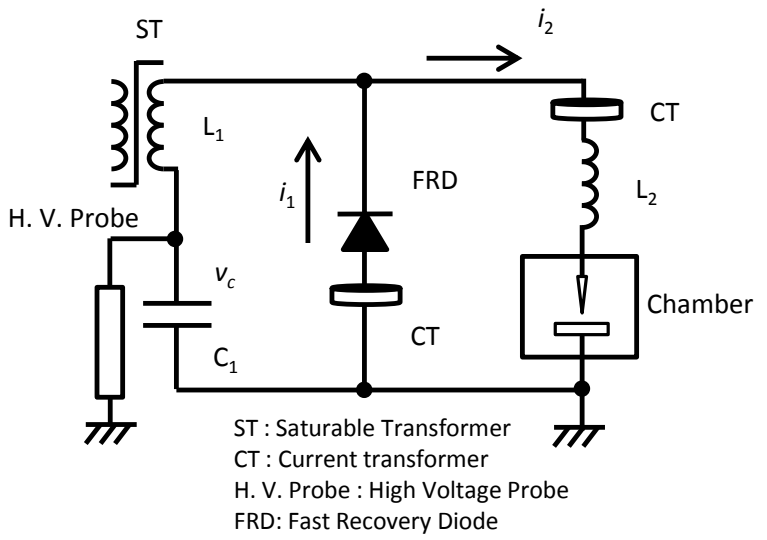

Fig. 3. Secondary circuit of inductive pulsed power generator.

measurement method was adopted due to its fast voltage rise rate $V_{r}$. The voltage drop at $L_{2}$ must be eliminated to measure the accurate applied voltage $v$ between the needle and plane electrode. $L_{2}$ is preliminarily calculated by dumped oscillation current by a short circuit method [7]. The $v$ can be calculated according to the following equation:

$$
v=L_{1} \frac{d i_{1}}{d t}+v_{c}-L_{2} \frac{d i_{2}}{d t}
$$

where $i_{1}$ is the current at the primary loop circuit including FRD, capacitor $\mathrm{C}_{1}(1 \mathrm{nF})$ and $\mathrm{L}_{1}$, which continuously flows until the FRD reverse current is discontinued, $i_{2}$ is the current for the load gap and begins to flow at the moment the FRD is opened, and $v_{\mathrm{c}}$ is the voltage of $\mathrm{C}_{1}$. Current and $v_{\mathrm{c}}$ were measured by current monitors (MODEL 6585, Pearson Electronics Inc. USA) and a high voltage probe (EP-100K, Nissin Pulse Co., Ltd. Japan). In the case of this equipment, the value of $v_{\mathrm{c}}$ needs approximately $4 \mathrm{kV}$ to obtain the maximum output voltage of $v$.

The magnetic pulse compression (MPC) circuit was also tested to examine the effect of different voltage rise times on pre-breakdown phenomena. The circuit of MPC was described in the previous paper [20]. The voltage was measured using a high voltage probe (EP-100K).

\section{RESULTS}

\section{A. Waveform of the applied voltage}

The applied voltage of IPPG calculated by the equation (1) is shown in Fig. 4. The time between 10 to $90 \%$ of voltage peak is termed voltage rise time. The voltage is characterized by the

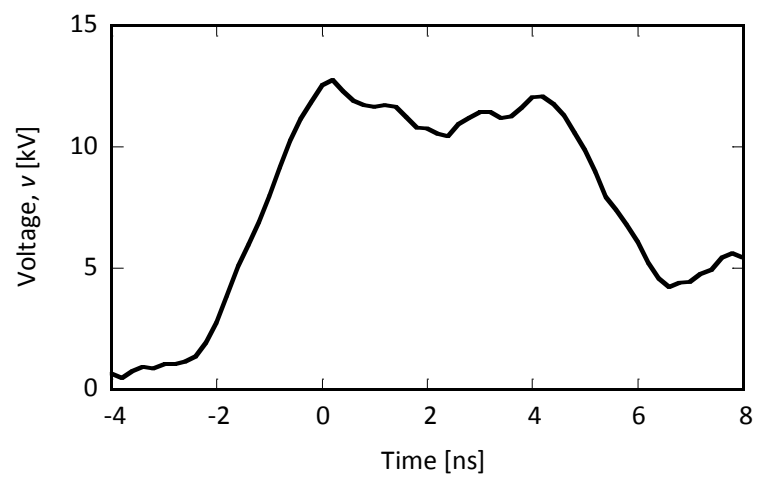

Fig. 4. Typical voltage waveform with voltage rise rate of $4.5 \mathrm{kV} / \mathrm{ns}$. 


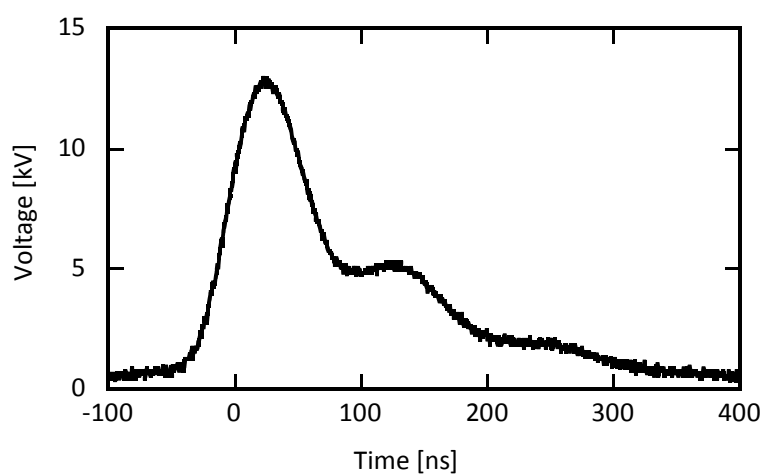

Fig. 5. Typical voltage waveform with voltage rise rate of $0.21 \mathrm{kV} / \mathrm{ns}$.

Table I. Comparison of the voltage parameters between inductive pulsed power generator (IPPG) and magnetic pulse compression (MPC) circuits.

\begin{tabular}{c|c|c}
\hline $\begin{array}{c}\text { Pulsed power } \\
\text { generator }\end{array}$ & Pulse-width $[\mathrm{ns}]$ & Voltage rise rate $[\mathrm{kV} / \mathrm{ns}]$ \\
\hline IPPG & 7.2 & 4.5 \\
\hline MPC & 77 & 0.21 \\
\hline
\end{tabular}

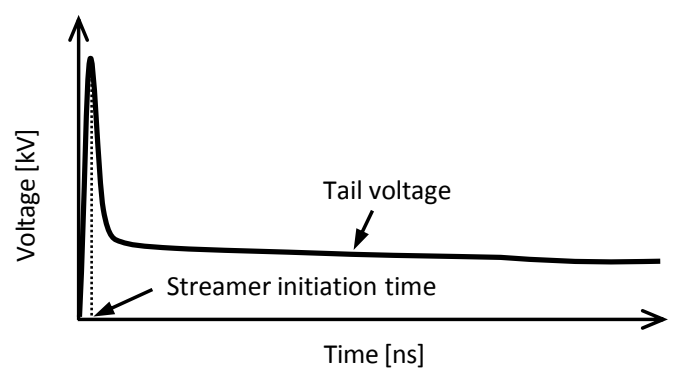

(a) IPPG

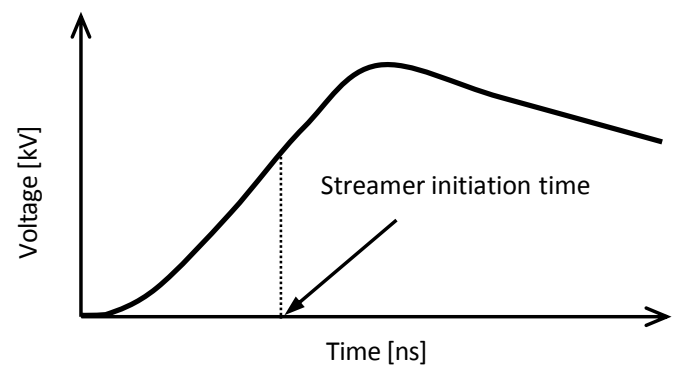

(b) MPC

Fig. 6. Illustration of two kinds of tested applied voltages: (a) Inductive pulsed power generator; and (b) magnetic pulse compression pulsed power generator.

fast $\mathrm{V}_{\mathrm{r}}=4.5 \mathrm{kV} / \mathrm{ns}$. A typical voltage waveform of MPC circuit is shown in Fig. 5. The slower $\mathrm{V}_{\mathrm{r}}=0.21 \mathrm{kV} / \mathrm{ns}$ was measured compared with IPPG. Each peak voltage was uniform at $13 \mathrm{kV}$. Pulse widths and $\mathrm{V}_{\mathrm{r}}$ of IPPG and MPC circuit are summarized in Table 1. An illustration of applied voltages with the IPPG and MPC circuit is shown in Fig. 6. The IPPG gave a relatively long residual voltage due to time constant of the circuit including large $\mathrm{C}_{1}$ following the ultra-short pulsed voltage, as seen in Fig. 6 (a). We refer to the residual voltage as "tail voltage". The initial value of the tail voltage and its time constant are $4 \mathrm{kV}$ and 1.6 second, respectively.

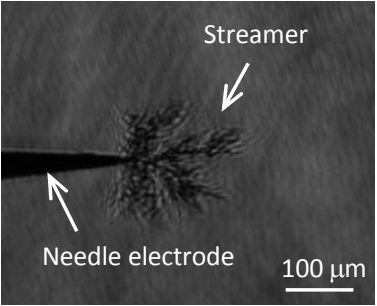

(a) $\mathrm{t}=23 \mathrm{~ns}$

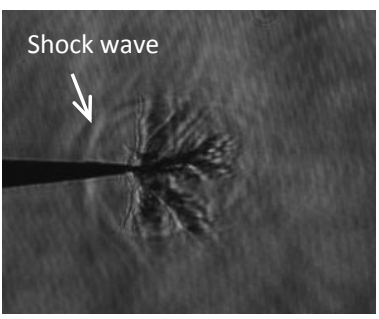

(c) $\mathrm{t}=320 \mathrm{~ns}$

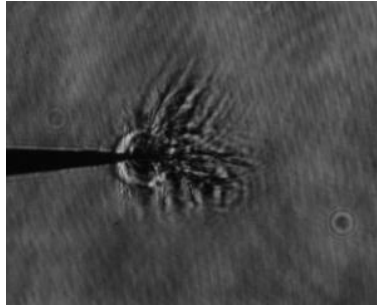

(b) $\mathrm{t}=119 \mathrm{~ns}$

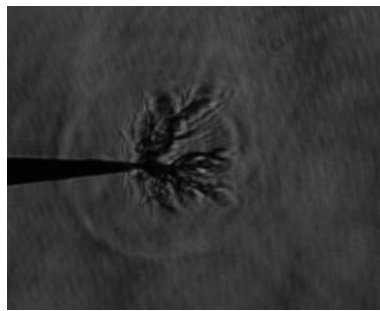

(d) $\mathrm{t}=450 \mathrm{~ns}$
Fig. 7. Shadowgraph images of pulsed discharge using inductive pulsed power generator with voltage rise rate of $4.5 \mathrm{kV} / \mathrm{ns}$ at positive peak voltage of $13 \mathrm{kV}$.

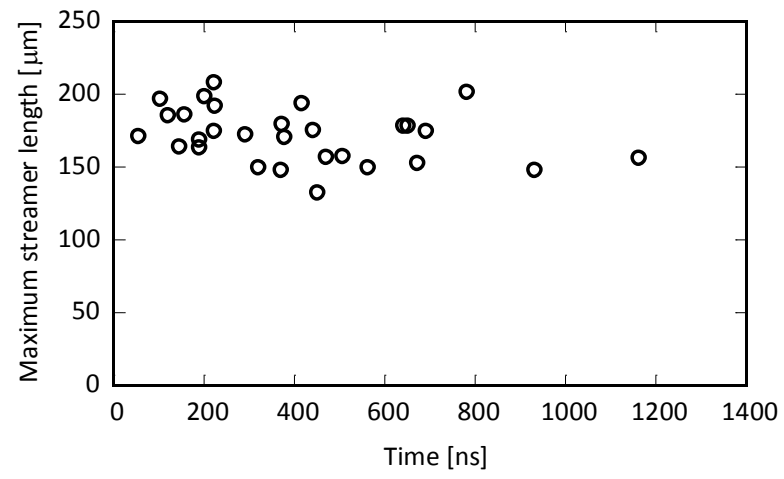

Fig. 8. Maximum streamer length as a function of time with voltage rise rate of $4.5 \mathrm{kV} / \mathrm{ns}$ at positive peak voltage of $13 \mathrm{kV}$.

\section{B. Observation results of pre-breakdown phenomena by means of shadowgraph method}

The streamer initiation time is defined as when the applied voltage reaches peak voltage due to its fast $\mathrm{V}_{\mathrm{r}}=4.5 \mathrm{kV} / \mathrm{ns}$, as shown in Fig. 6 (a). Shadowgraph images of pre-breakdown phenomena with $\mathrm{V}_{\mathrm{r}}=4.5 \mathrm{kV} / \mathrm{ns}$ are shown in Fig. 7. A multiple-branched streamer grows in several directions, and shock waves propagate from the streamer. Here, there is a concern about the influence of the tail voltage on streamer growth. The maximum streamer length is almost constant irrespective of the time lapse up to $1200 \mathrm{~ns}$ from streamer initiation, as shown in Fig. 8. Thus, the influence of the tail voltage on streamer growth is negligible. When the slower $V_{r}=$ $0.21 \mathrm{kV} / \mathrm{ns}$ is applied, the streamer initiates with current pulse on the occasion of voltage rise, as shown in Fig. 6 (b). Shadowgraph images of pre-breakdown phenomena with $\mathrm{V}_{\mathrm{r}}=$ $0.21 \mathrm{kV} / \mathrm{ns}$ are shown in Fig. 9. The streamer length with MPC $(t \sim 12$ ns, see Fig. 9 (a)) was shorter than with the IPPG (see Fig. 7). The low streamer initiation voltage under MPC may influence the short streamer length at the initial stage. A 


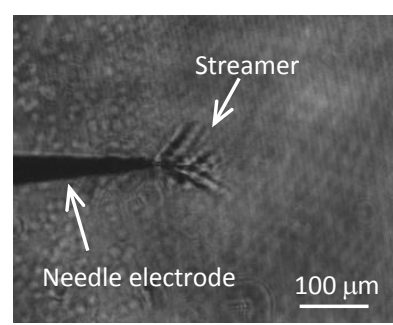

(a) $\mathrm{t}=12 \mathrm{~ns}$

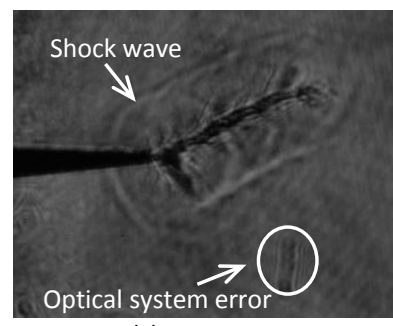

(c) $\mathrm{t}=322 \mathrm{~ns}$

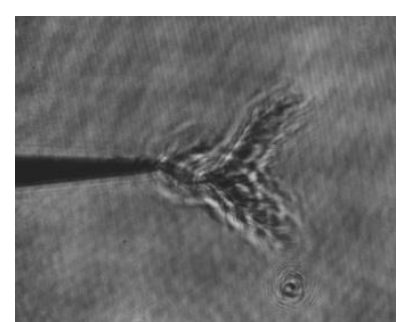

(b) $t=124 n s$

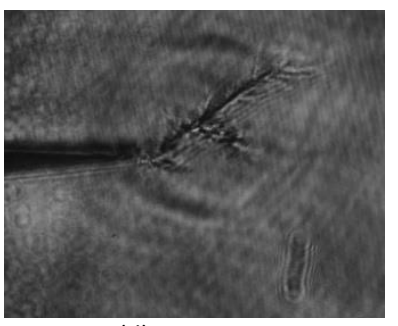

(d) $t=472 \mathrm{~ns}$
Fig. 9. Shadowgraph images of pulsed discharge using magnetic pulse compression circuit with voltage rise rate of $0.21 \mathrm{kV} / \mathrm{hs}$ at positive peak voltage of $13 \mathrm{kV}$.

streamer with fewer branches grows, and shock waves propagate along the streamer channel. A comparison of the streamers channel between Fig. 7 and Fig. 9 shows that streamer channel diameter increases with increasing $\mathrm{V}_{\mathrm{r}}$.

\section{DISCUSSION}

\section{A. Positive streamer branching with different voltage rise rate}

No density change due to such as electrostrictive force by slower $\mathrm{V}_{\mathrm{r}}$ was detected under $\mathrm{SC}-\mathrm{CO}_{2}$ in our previous study [17]. Recently, nano-sized pore generation in dielectric liquids by ponderomotive electrostriction effect was supposed under sub-nanosecond voltage rise time [14]-[16]. Therefore, the possibility of density change should be discussed under the fast $\mathrm{V}_{\mathrm{r}}$. According to [14], volumetric pondermotive (electrostrictive) force $F$ for liquids can be classified into nonpolar and polar liquids as follows:

Clausius-Mosotti formula for nonpolar dielectrics

$$
\vec{F}=\frac{\varepsilon_{0}}{2} \nabla\left(E^{2} \frac{\partial \varepsilon}{\partial \rho} \rho\right)=\frac{\varepsilon_{0}}{2} \frac{(\varepsilon-1)(\varepsilon+2)}{3} \nabla E^{2}
$$

and for polar dielectrics

$$
\vec{F}=\frac{\varepsilon_{0}}{2} \nabla\left(E^{2} \frac{\partial \varepsilon}{\partial \rho} \rho\right) \approx \xi \varepsilon_{0} \varepsilon \nabla E^{2} \quad(\xi \approx 1)
$$

where $\varepsilon_{0}$ is the vacuum permittivity, $\varepsilon$ is the relative dielectric constant of the medium, $E$ is the field vicinity of the needle tip, $\rho$ is the density of the medium. Relative permittivity of $\mathrm{CO}_{2}$ drastically changes as a function of medium density. In our experimental condition ( $305 \mathrm{~K}, 7.5 \mathrm{MPa})$, relative permittivity was estimated as approximately 1.2 [21]. In the case of $\mathrm{SC}-\mathrm{CO}_{2}$, the equation (2) is adopted due to the nonpolar molecularity of $\mathrm{CO}_{2}$. Under conditions of distilled water with relative permittivity of 80 [15], the equation (3) is adopted due to the polar molecularity of water. The ratio of volumetric

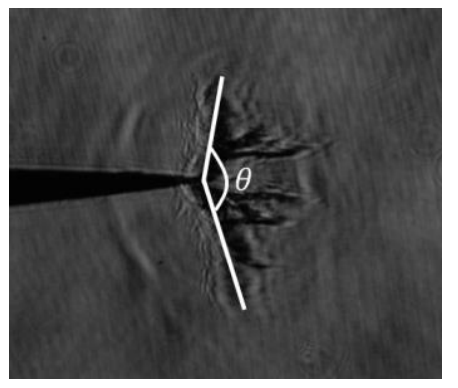

Fig. 10. Determination of branching streamer spread angle with voltage rise rate of $4.5 \mathrm{kV} / \mathrm{ns}$ at positive peak voltage of $13 \mathrm{kV}(\mathrm{t}=415 \mathrm{~ns})$.

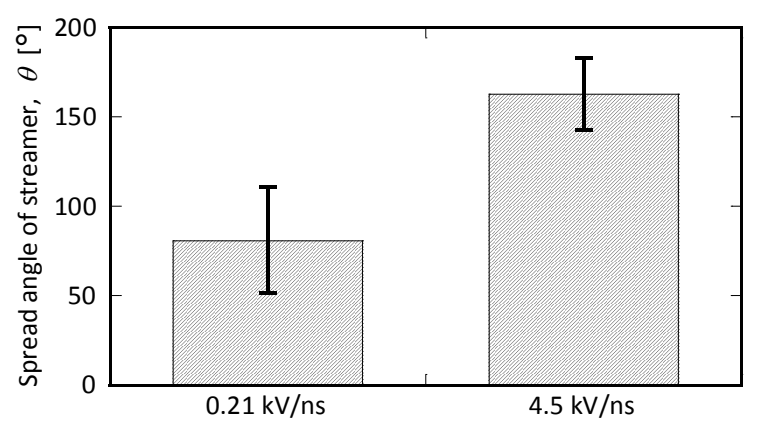

Fig. 11. Spread angle of the positive streamer branching under different voltage rise rate at positive peak voltage of $13 \mathrm{kV}$.

electrostrictive force under $\mathrm{SC}-\mathrm{CO}_{2}\left(F_{\mathrm{SC}}\right)$ to distilled water $\left(F_{\text {water }}\right)$ is 0.001 . The small value of $F_{\mathrm{SC}} / F_{\text {water }}$ assists in assumption of no bubble generation in $\mathrm{SC}-\mathrm{CO}_{2}$ but the value of 0.001 does not refer to the threshold of bubble generation by field intensity. On the other hand, bubble generation in discharge initiation process is not expected due to the nonexistence of evaporative latent heat under SC condition. Consequently, effect of density change at the needle tip by electrostrictive force is negligible in $\mathrm{SC}-\mathrm{CO}_{2}$ and has an insignificant effect on the streamer initiation.

A typical shadowgraph image of positive streamer discharge with $\mathrm{V}_{\mathrm{r}}=4.5 \mathrm{kV} / \mathrm{ns}$ is shown in Fig. 10. The spread angle of the streamer at the needle tip is defined as $\theta$. The measured $\theta$ strongly depends on $\mathrm{V}_{\mathrm{r}}$ as shown in Fig. 11. Both $\theta$ are the mean values of 30 measurements, and the error bar indicates standard deviation. Streamers with fewer branches due to the slower $\mathrm{V}_{\mathrm{r}}$ suppose a moderate field change at the needle tip that induces field enhancement at the most likely pre-breakdown spot. Other viable streamers at the needle tip are relatively weak due to the growth of the streamer. By contrast, the multiple-branched streamer with faster $V_{r}$ is able to grow from the needle tip in several directions simultaneously due to rapid field change. Furthermore, streamer initiation voltage may affect the streamer branching due to the dependence of streamer initiation voltage on $\mathrm{V}_{\mathrm{r}}$. A positive correlation between corona inception voltage and $\mathrm{V}_{\mathrm{r}}(0.5-3 \mathrm{kV} / \mathrm{ns})$ was reported in [22]. The identical effect of the initiation voltage on $\mathrm{V}_{\mathrm{r}}$ could be inferred in $\mathrm{SC}-\mathrm{CO}_{2}$ because the initiation mechanism of positive streamer in $\mathrm{SC}-\mathrm{CO}_{2}$ is suggested a direct ionization process [18]. Consequently, it is supposed that a number of initial electrons are drifted to the needle tip by rapidly enhanced field of IPPG. 


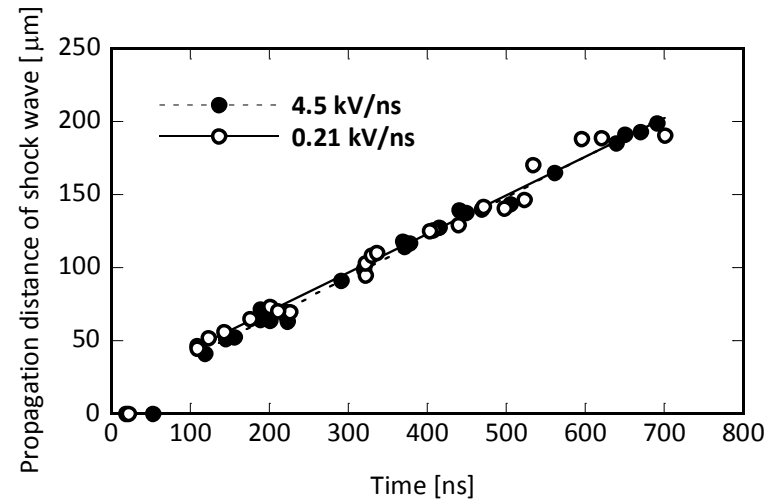

Fig. 12. Propagation distance of shock wave as a function of time under different voltage rise times at positive peak voltage of $13 \mathrm{kV}$.

\section{B. Behavior of shock wave propagation}

The propagation distance of shock waves between the positive streamer channel and the shock front was measured as a function of time, as shown in Fig. 12. Streamer initiation time defined as stated in section III-B corresponds to $t=0$. The propagation distance of shock waves remains mostly constant irrespective of different $V_{r}$. The delay time before shock wave initiation, discussed as the relaxation time of vibration energy by the experiment of negative pulsed discharge in $\mathrm{SC}-\mathrm{CO}_{2}$ in a previous paper, was up to $t \sim 100 \mathrm{~ns}$ in both $\mathrm{V}_{\mathrm{r}}$ [17], [23]. A corresponding mechanism may be supposed in the case of the positive discharge. Mach numbers under $\mathrm{V}_{\mathrm{r}}=0.21$ and 4.5 $\mathrm{kV} / \mathrm{ns}$ are 1.57 and 1.64 , respectively, where the sound speed is based on literature [24]. However, Mach number shows little dependence on $V_{r}$, as shown in Fig. 12. Thus, the variation of $V_{r}$ in the range of this study has little effect on Mach number.

Meanwhile, characteristic of propagation distances of shock waves by different negative voltage peaks were investigated to confirm effects of the voltage peak on the shock wave. The negative polarity was adopted because the positive discharge easily breaks down when the voltage increases up to a few tens kilovolt, and the same shock wave generation mechanism is assumed irrespective of polarity of streamer because of both subequal delay times from streamer initiation to shock wave generation. The MPC circuit was used for this experiment since the negative peak voltage could be drastically changed. Appearance of bush-like negative streamer with spherical shock wave under different negative voltage peak is shown in Fig. 13. Clearer shock waves were observed under $60 \mathrm{kV}$ (Fig. 13 (b)) than under $40 \mathrm{kV}$ (Fig. 13 (a)). Shock wave propagation distances under different negative voltage peaks are shown in Fig. 14. The propagation distance of shock wave was determined between needle tip and shock front. The vertical shift between 40 and $60 \mathrm{kV}$ in Fig. 14 was influenced by the size of negative streamer. The negative streamer initiation voltage was calculated based on a field emission [17] corresponding to $t=0$. Mach number with peak voltages of 40 and $60 \mathrm{kV}$ are 1.54 and 1.70, respectively. The Mach number under $60 \mathrm{kV}$ is 1.1 times greater than that under $40 \mathrm{kV}$. As stated above, the shock wave generation mechanism with negative voltage can be explained by the release of stored

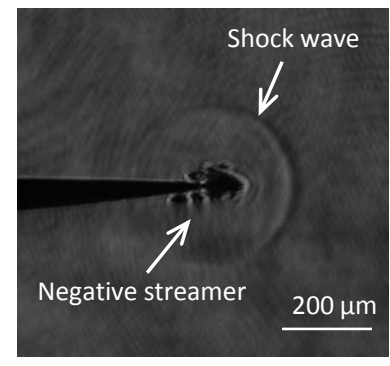

(a) $40 \mathrm{kV}, \mathrm{t}=609 \mathrm{~ns}$

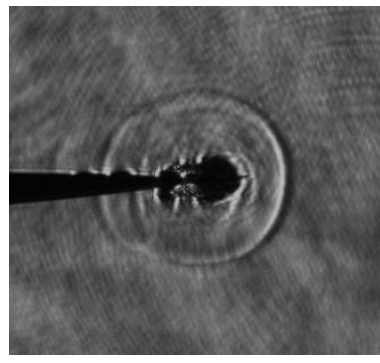

(b) $60 \mathrm{kV}, \mathrm{t}=602 \mathrm{~ns}$
Fig. 13. Shadowgraph images of negative pulsed discharge. (a) $40 \mathrm{kV}, \mathrm{t}=609$ ns. (b) $60 \mathrm{kV}, \mathrm{t}=602 \mathrm{~ns}$.

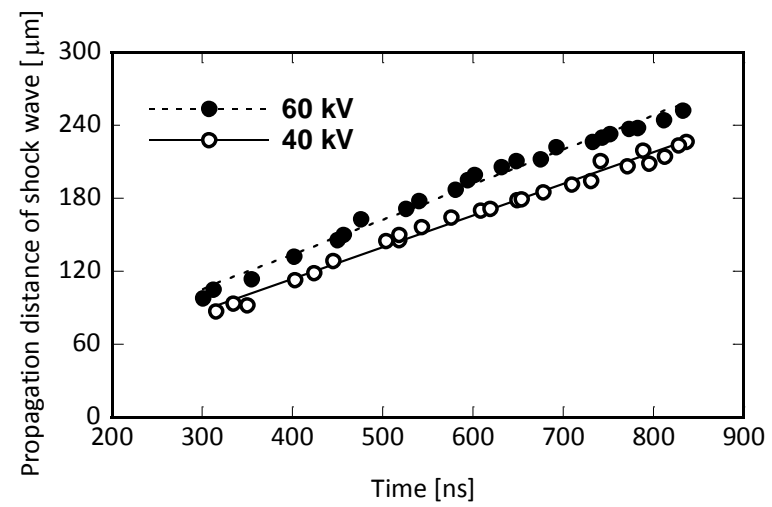

Fig. 14. Propagation distance of shock wave as a function of time under different negative voltage peaks of 40 and $60 \mathrm{kV}$.

vibrational energy. The increase of electron collision rate by an increase in voltage peak may affect the population of vibrational state $\mathrm{CO}_{2}$ because the size of negative streamer increased with increasing applied negative voltage as shown in Fig. 13.

\section{CONCLUSION}

The pre-breakdown process of positive discharge in a non-uniform field in $\mathrm{SC}-\mathrm{CO}_{2}$ was observed by means of the shadowgraph method. Different voltage rise rates $V_{r}$ of 4.5 and $0.21 \mathrm{kV} / \mathrm{ns}$ were applied to the needle electrode. Streamer spread angles and shock wave Mach numbers were discussed. The results can be summarized as follows.

1) The primary filament-like positive streamer initiates from the needle electrode. The streamer channel diameter increases with increasing $\mathrm{V}_{\mathrm{r}}$. Moreover, streamer spread angle under $4.5 \mathrm{kV} / \mathrm{ns}$ at the needle tip is twice as large as that under $0.21 \mathrm{kV} / \mathrm{ns}$. Although discharge initiates without density reduction due to electrostrictive force, $\mathrm{V}_{\mathrm{r}}$ affects the spread of the positive streamer.

2) The shock wave propagates vertically from the positive streamer channel. Delay times from streamer initiation to shock wave initiation exist on the order of a few tens nanoseconds. The Mach number is independent of $V_{r}$ but dependent on negative voltage peak. This dependence on voltage peak is believed to be due to an increase in the population of vibrational state $\mathrm{CO}_{2}$. 


\section{ACKNOWLEDGMENT}

The authors would like to thank T. Imamichi, master course student, for his assistance in the experiments. This work was supported by a Grant-in-Aid for Research Activity Start-up (No. 26889048) and by TEPCO Memorial Foundation.

\section{REFERENCES}

[1] T. Kiyan, M. Sasaki, T. Ihara, T. Namihira, M. Hara, M. Goto, and H. Akiyama, "Pulsed breakdown and plasma-aided phenol polymerization in supercritical carbon dioxide and sub-critical water," Plasma Process. Polym., vol. 6, no. 11, pp. 778-785, Nov. 2009.

[2] A. Kawashima, S. Nomura, H. Toyota, T. Takemori, S. Mukasa and T. Maehara, "A supercritical carbon dioxide plasma process for preparing tungsten oxide nanowires," Nanotechnology, vol. 18, no. 49, p. 495603 , Dec. 2007.

[3] T. Ito, K. Katahira, Y. Shimizu, T. Sasaki, N. Koshizaki, and K. Terashima, "Carbon and copper nanostructured materials syntheses by plasma discharge in a supercritical fluid environment," J. Mater. Chem., vol. 14, no. 10, pp. 1513-1515, Apr. 2004.

[4] S. Nakahara, S. Stauss, T. Kato, T. Sasaki, and K. Terashima, "Synthesis of higher diamondoids by pulsed laser ablation plasmas in supercritical $\mathrm{CO}_{2}$," J. Appl. Phys., vol. 109, no. 12, pp. 123304-1-123304-8, Jun. 2011.

[5] J. Zhang, B. van Hessch, F. Beckers, T. Tuiskamp, and G. Pemen, "Breakdown Voltage and Recovery Rate Estimation of a Supercritical Nitrogen Plasma Switch," IEEE Trans. Plasma Sci., vol. 42, no. 2, pp. 376-383, Feb. 2014.

[6] Z.B. Yang, S. H. R. Hosseini, T. Kiyan, S. Gnapowski, and H. Akiyama, "Post-breakdown dielectric recovery characteristics of high-pressure liquid $\mathrm{CO}_{2}$ including supercritical phase," IEEE Trans. Dielectr. Electr. Insul., vol. 21, no. 3, pp. 1089-1094, Jun. 2014.

[7] H. Tanoue, T. Furusato, T. Imamichi, M. Ota, S. Katsuki, and H. Akiyama, "Dielectric recovery mechanism of pressurized carbon dioxide at liquid and supercritical phases," Jpn. J. Appl. Phys., vol. 54, no. 9, pp. 096102-1-096102-8, Aug. 2015.

[8] H. Muneoka, K. Urabe, S. Stauss, and K. Terashima, "Breakdown Characteristics of Electrical Discharges in High-Density Helium Near the Critical Point," Appl. Phys. Express, vol. 6, no. 8, 086201-1-086201-4, Aug. 2013.

[9] H. Muneoka, K. Urabe, S. Stauss, and K. Terashima, "Micrometer-scale electrical breakdown in high-density fluids with large density fluctuations: Numerical model and experimental assessment," Phys. Rev. E, vol. 91, no. 4, pp. 042316-1-042316-11, Apr. 2015.

[10] T. Kiyan, A. Uemura, B. C. Roy, T. Namihira, M. Hara, M. Sasaki, M. Goto, and H. Akiyama, "Negative DC Pre-breakdown Phenomena and Breakdown Voltage Characteristics of Pressurized Carbon Dioxide up to Supercritical Conditions," IEEE Trans. Plasma Sci., vol. 35, no. 3, pp. 656-662, Jun. 2007.

[11] T. Kiyan, M. Takade, T. Namihira, M. Hara, M. Sasaki, M. Goto, and H. Akiyama, "Polarity Effect in DC Breakdown Voltage Characteristics of Pressurized Carbon Dioxide up to Supercritical Conditions," IEEE Trans. Plasma Sci., vol. 36, no. 3, pp. 821-827, Jun. 2008.

[12] J. F. Kolb, P. R. Joshi, S. Xiao, and K. H. Schenbach, "Streamer in water and other dielectric liquids," J. Phys. D, Appl. Phys., vol. 41, no. 23, pp. 234007-1-234007-22, Dec. 2008.

[13] A. Wladimir, K. Baumung, and H. Bluhm, "Underwater streamer propagation analysis from detailed measurements of pressure release," $J$. Appl. Phys., vol. 101, no. 5, pp. 053302-1-053302-10, Mar. 2007.

[14] M. N. Shneider, M. Pekker, and A. Fridman, "Theoretical Study of the Initial Stage of Sub-nanosecond Pulsed Breakdown in Liquid Dielectrics," IEEE Trans. Dielectr. Electr. Insul., vol. 19, no. 5, pp. 1579-1582, Jul. 2012.

[15] D. Dobrynin, Y. Seepersad, M. Pekker, M. Shneider, G. Friedman, and A. Fridman, "Non-equilibrium nanosecond-pulsed plasma generation in the liquid phase (water, PDMS) without bubbles: fast imaging, spectroscopy and leader-type model," J. Phys. D: Appl. Phys., vol. 46, no. 10, pp. 105201-1-105201-9, Mar. 2013.

[16] Y. Seepersad, M. Pekker, M. Shneider, D. Dobrynin, and A. Fridman, "On the electrostrictive mechanism of nanosecond-pulsed breakdown in liquid phase," J. Phys. D: Appl. Phys., vol. 46, no. 16, pp. 162001-1-162001-6, Apr. 2013.

[17] T. Furusato, T. Ihara, T. Kiyan, S. Katsuki, M. Hara, and H. Akiyama, "Initiation mechanism of a negative nanosecond pulsed discharge in supercritical carbon dioxide," IEEE Trans. Plasma Sci., vol. 40, no. 11, pp. 3105-3115, Nov. 2012.

[18] T. Ihara, T. Furusato, S. Kameda, T. Kiyan, S. Katsuki, M. Hara, and H. Akiyama, "Initiation mechanism of positive streamer in pressurized carbon dioxide up to liquid and supercritical phases with nanosecond pulsed voltages," J Phys. D, Appl. Phys., vol. 45, no. 7, pp. 075204-1075204-10, Feb. 2012.

[19] T. Furusato, H. Tanoue, S. Katsuki, M. Hara, and H. Akiyama, "Prebreakdown process of a negative nanosecond pulsed discharge in supercritical carbon dioxide," Appl. Phys. Express, vol. 7, no. 2, pp. 026201-1- 026201-3, Jan. 2014.

[20] T. Furusato, T. Ihara, S. Kameda, T. Kiyan, S. Katsuki, M. Hara, and H. Akiyama, "Visualization of positive pulsed streamer in supercritical carbon dioxide by Schlieren method," in Proc. 18th IEEE Int. Pulsed Power Conf., Chicago, IL, 2011, pp. 280-284.

[21] T. Moriyoshi, T. Kita, and Y. Uosaki, "Static Relative Permittivity of Carbon Dioxide and Nitrous Oxide up to $30 \mathrm{MPa}$," Ber. Bunsenges. Phys. Chem., vol. 97, no. 4, pp. 589-596, 1993.

[22] A. Fridman, "Plasma Chemistry," Cambridge University Press, pp. 234-235, 2008.

[23] H. Tanoue, T. Furusato, K. Takahashi, S. H. R. Hosseini, S. Katsuki and H. Akiyama, "Characteristics of Shock Waves Generated by a Negative Pulsed Discharge in Supercritical Carbon Dioxide," IEEE Trans. Plasma Sci., vol. 42, no. 10, pp. 3258-3263, Oct. 2014.

[24] R. Span and W. Wagner, "A new equation of state for carbon dioxide covering the fluid region from the triple-point temperature to $1100 \mathrm{~K}$ at pressures up to $800 \mathrm{MPa}$," J. Phys. Chem. Ref. Data, vol. 25, no. 6, pp. 1509-1596, Nov. 1996.

Tomohiro Furusato (S'13-M'14) was born in Kagoshima, Japan, in 1988. He received the B. E., M. E., and D. E. degrees from Kumamoto University, Kumamoto Japan, in 2011, 2012, and 2014, respectively. He was with the Japan Society for the Promotion of Science, Kumamoto University, from 2013 to 2014, as a Research Fellow. Since 2014, he has been an Assistant Professor with the Graduate School of Engineering, Nagasaki University, Nagasaki, Japan. His research interests are pulsed-power, and discharge phenomena in supercritical fluids.

Miyuki Ota was born in Kumamoto, Japan, in 1991. She received B.E and M.E degree in 2014 and 2016 respectively from Kumamoto University, Kumamoto, Japan. Her current research interests are discharge phenomena in supercritical fluid and bioelectrics.

Tomoyuki Fujishima was born in 1966 at Fukuoka in Japan. He received the B.E., M.E. and D.E. degrees in electrical engineering from Kumamoto University, Japan, in 1989, 1991 and 1994, respectively. He has been working in Nagasaki University since 1994 and is an Associate Professor of the Graduate School of Engineering, Nagasaki University, Japan. His research interests are ozone generation, ozonic environment application and lightning protection. He is a member of the Institute of Electrical Engineers of Japan and the Japan Society of Applied Physics.

Takahiko Yamashita (M'00) was born in 1957 at Fukuoka in Japan. He received the B. E., M. E. and D. E. degrees from Kyushu University in 1980, 1982 and 1985, respectively. He has been working in Nagasaki University, Nagasaki, Japan since 1985. He is a professor of Graduate School of Engineering and a Vice President of Nagasaki University. He is a senior member of Institute of Electrical Engineers Japan. 
Takashi Sakugawa (M'04) received the M.E. degree from Kyushu University, Fukuoka, Japan, in 1989, and the Ph.D. degree from Kumamoto University, Kumamoto, Japan, in 2004. He was with the Central Research Laboratory, Meidensha Corporation, Tokyo, Japan, from 1989 to 2004. From 2004 to 2008, he was an Associate Professor with the Corporative Research Center, Kumamoto University, where he has been a Professor with the Institute of Pulsed Power Science.

Sunao Katsuki (M'99) was born in Kumamoto, Japan, in 1966. He received the B.S., M.S., and Ph.D. degrees from Kumamoto University, Kumamoto, Japan, in 1989, 1991, and 1998 respectively. He became a Research Associate at Kumamoto University in 1991. From 1998 to 2006 he had been an Associate Professor in the Department of Electrical and Computer Engineering, Kumamoto University. During 2001-2002, he was a Senior Researcher at Old Dominion University, USA. Since 2007, he has been a Director of Bioelectrics Research Center, Kumamoto University. His research interests are pulsed power, bioelectrics, underwater discharges and Z-pinch plasmas. He is a member of IEEE, The Institute of Electrical Engineers of Japan, and The Japan Society of Plasma Science and Nuclear Fusion Research.
Hidenori Akiyama (M'87-SM'99-F'00) received the Ph.D. degree from Nagoya University, Nagoya, Japan, in 1979. He was a Research Associate with Nagoya University from 1979 to 1985 , and since then, he has been on the faculty of Kumamoto University, Kumamoto, Japan. He was a Director of the 21st Century Center of Excellence (COE) program on pulsed power science and is a Director of the global COE program on pulsed power engineering, which is supported by the Ministry of Education, Culture, Sport, Science and Technology, Japan, for ten years. He is the author of about 250 refereed journal papers and ten books. Dr. Akiyama was the recipient of the "IEEE Major Educational Innovation Award, 2000, " "IEEE Peter Haas Award, 2003," "IEEE Germeshausen Award, 2008," and "Frank Raidy Bioelectrics Award, 2011. 